\title{
Mucociliary transport in trachea of patients with cystic fibrosis
}

\author{
DONOVAN B. YEATES, JENNIFER M. STURGESS, SHAHIDA R. KAHN, \\ HENRY LEVISON, AND NORMAN ASPIN \\ From the Departments of Medical Biophysics, Pathology, and Paediatrics, University of Toronto, and the \\ Research Institute, Hospital for Sick Children, Toronto, Canada
}

\begin{abstract}
Yeates, D. B., Sturgess, J. M., Kahn, S. R., Levison, H., and Aspin, N. (1976). Archives of Disease in Childhood, 51, 28. Mucociliary transport in trachea of patients with cystic fibrosis. Mucociliary tracheal transport rates were measured in 20 patients with cystic fibrosis, in whom these rates ranged from 0 to $12.8 \mathrm{~mm} / \mathrm{min}$. The patients were divided into 3 roughly equal groups on the basis of their transport rates. (1) Those in whom no abnormality in mucociliary transport was detected in the trachea; (2) those in whom normal transport rates were measured but in whom abnormalities such as cessation, or reversal of bolus movement were observed; (3) those in whom no normal transport rates were observed. In the first group the rates were similar to those observed in a population of healthy adults. These normal rates were observed in some patients who had a productive cough. The mean mucociliary tracheal transport rate increased with increasing maximum midexpiratory flow. Those patients with a low Shwachman score and poor arterial oxygen tension tended to fall into groups 2 and 3 . In the ciliary dyskinesia assay in rabbit trachea the serum from the patients with the higher transport rates tended to initiate more rapid discharge of material from the epithelium and ciliary dyskinesia.
\end{abstract}

A defect in the mucociliary transport mechanism has been implicated in the pathogenesis of lung disease in cystic fibrosis (CF). This hypothesis has resulted from several observations. Patients with CF secrete more mucus than normal subjects, as indicated by gland hypertrophy (Zuelzer and Newton, 1949; Reid and de Haller, 1967; Esterly and Oppenheimer, 1968), and frequently retain secretions in the lung (Zuelzer and Newton, 1949; Esterly and Oppenheimer, 1968). This mucus has been reported to have altered biochemical (Matthews et al., 1963; Potter et al., 1963) and rheological (Denton, 1960) properties. While the physiochemical properties of mucus are altered in lung disease, little difference has been shown in histochemical (Lamb and Reid, 1972) or rheological (Sturgess and Reid, 1969; Charman and Reid, 1972; Feather and Russell, 1970) studies between mucus from patients with CF and patients with other hypersecretory conditions such as chronic bronchitis or bronchiectasis. A factor in the serum

Received 21 April 1975. of CF patients inhibits normal ciliary activity in explants of ciliated epithelium (Spock, Heick, and Logan, 1967; Conover et al., 1973). In addition, poor mucociliary transport could result from infection (Camner, Jarstrand, and Philpson, 1973a, b; Sakakura et al., 1973) which damages human ciliated epithelium in culture (Hoorn and Tyrrell, 1966). Recent studies report conflicting results regarding the transport of particles from the lungs of CF patients (Sanchis et al., 1973; Thomson et al., 1974; Wood, Wanner, and Hirsch, 1974).

This study was designed to determine whether an abnormality in mucociliary transport was present in the trachea of CF patients and to investigate whether the measured transport rates were related to the ciliary dyskinesia factor in their serum.

\section{Methods}

Mucociliary tracheal transport rates (MTTRs) were measured on $20 \mathrm{CF}$ patients (12 males, 8 females) aged 11-20 years. Informed consent to perform these studies was obtained both from the patients and their parents. The experimental protocol was approved by 
the Human Experimentation Committee at the University of Toronto. MTTR was measured by two methods: 4 subjects were measured in the upright position and 18 in the supine position.

MTTRs. The first group of patients was studied in the upright position using the method of Yeates et al. (1975). By this method an aqueous aerosol containing albumin microspheres $(0.5 \mu \mathrm{m}$ count median diameter) labelled with ${ }^{99} \mathrm{Tcm}$ (Yeates, Warbick, and and Aspin, 1974a) was inhaled so that it was deposited in local concentrations in the large airways. $15 \mathrm{~min}$ utes before the study each subject was given potassium perchlorate $(8 \mathrm{mg} / \mathrm{kg}$ ) to inhibit the uptake of free pertechnetate by the thyroid gland. The bolus of radioactive microspheres was transported up the trachea and its position recorded with a gamma camera. The MTTR of each bolus was determined from these measurements.

In the second group of patients, MTTR was measured in the supine position using a scanning scintillation probe. The patients inhaled the labelled aerosol as described by Yeates et al. (1975) until a pair of scintillation probes positioned behind the subject's lungs indicated that sufficient radioactivity had been deposited. Then the subject immediately washed his mouth with water and lay on his back under the scintillation probe. The position of the trachea with respect to the scanner was determined using a point source of radioactivity placed over the carina (position determined from a chest $x$-ray) and the larynx. The alignment of the subject with respect to the probe was checked at regular intervals using markers on the subject's skin and on the scanning probe. To ensure that the activity measured by the probe was in the trachea and not in the oesophagus the subject was asked to drink water at regular intervals. The probe, which had a parallel slit collimator, scanned back and forth along the trachea at a rate of $19 \mathrm{~cm} / \mathrm{min}$. The activity measured by this probe was recorded using a rate meter connected to a chart recorder. The time of occurrence and the forcefulness of every cough was recorded. Each subject was scanned for a period of 2 hours.

The position of each radioactive bolus in the trachea was plotted as a function of time and the transport rate determined using the method of least squares fit. A significant MTTR resulted when the position of the bolus was measured on at least four tracings of the profile of the radioactivity over the trachea. These must also represent movement of the bolus over at least $2.5 \mathrm{~cm}$ of the trachea or observation of the bolus for at least 15 minutes in time. Transport rates were analysed only during periods when the patient did not cough. An abnormal MTTR was defined as one which was zero or negative. When calculating the average MTTR a negative value was considered as a zero transport rate.

Clinical history. The following data were recorded for each subject: Shwachman score, chest $x$-ray, extent of cough and sputum production, and assessment of clinical condition at the time of the study together with the lung history over the past 2-5 years. Before each study static lung volumes were measured by plethysmography (DuBois et al., 1956), functional residual capacity, and total lung capacity, and maximal midexpiratory flow (MMEF) by water spirometry. Oxygen tension $\left(\mathrm{PaO}_{2}\right)$ and carbon dioxide tension $\left(\mathrm{PaCO}_{2}\right)$ were measured in duplicate specimens of arterialized capillary blood 10 minutes after the application of a vasodilator cream. Sputum cultures were made before and after the study.

Serum factor assay. Blood samples were collected from 14 patients about one year after completion of the MTTR study. The serum was separated and assayed for the ciliary dyskinesia factor using the method of Spock, Heick, and Logan (1967) as modified by Conover et al. (1973). These sera were measured in such a way that the observer was not aware of the magnitude of each patient's MTTR.

Explants of rabbit tracheal epithelium were incubated in tissue culture medium at $37^{\circ} \mathrm{C}$ in $5 \%$ carbon dioxide/ 95\% oxygen for 24-48 hours. Each explant was incubated with $0.2 \mathrm{ml}$ serum from a CF patient or from a control subject. The amount of the discharge of material from its surface and the alterations in ciliary activity were recorded at 5 -minute intervals over a period of 35 minutes.

\section{Results}

The patients' MTTRs, lung function tests, and results of serum factor assays are shown in the Table. The patients are listed according to their transport rates, the mean MTTRs ranging from $0-12 \cdot 8 \mathrm{~mm} / \mathrm{min}$. The patients are divided into three roughly equal groups: (1) those in whom no abnormal transport rate was detected (Cases 1-10), (2) those in whom positive tracheal transport rates were measured but who also showed abnormalities such as a radioactive bolus residing at one spot in the trachea for more than 15 minutes or a bolus moving distally in the trachea (Cases 11-16b), and (3) those in whom no normal tracheal transport was observed (Cases 16a-20). Cases 3 and 16 who had repeated tests one year apart, gave similar results on both. Of the patients with the three fastest rates, Cases 2 and 3 had essentially normal values of all parameters measured and did not cough up any mucus, and Case 1 , though his values were below normal, had normal results in his function tests at other times. It was remarkable that in Cases 4-8 and 10, who had productive coughs normal MTTRs were measured. In some patients the boli remained stationary or slid slowly backwards in the trachea, but were cleared subsequently by coughing.

The clinical factor with the highest correlation to mean MTTR was the MMFF $(0 \cdot 1<P<0 \cdot 5)$. 


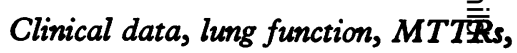

\begin{tabular}{|c|c|c|c|c|c|c|}
\hline $\begin{array}{l}\text { Case } \\
\text { no. }\end{array}$ & $\begin{array}{l}\text { Age } \\
\text { (yr) }\end{array}$ & $\begin{array}{l}\text { Shwachman } \\
\text { score }\end{array}$ & $\begin{array}{c}\text { MMEF } \\
\text { (\% predicted }\end{array}$ & $\begin{array}{c}\text { FRC/ } \\
\text { TLC } \\
(\%)\end{array}$ & $\underset{(\mathrm{mmHg})}{\mathrm{PaCO}_{2}}$ & $\underset{\left(\mathrm{mmH}_{2}\right.}{\mathrm{PaO}_{2}}$ \\
\hline 1 & 11 & 85 & 46 & 47 & 38 & 76 \\
\hline $2^{\star}$ & 15 & 80 & 124 & 37 & 38 & 90 \\
\hline $3 * a$ & 12 & 95 & 137 & 45 & 36 & 81 \\
\hline $\begin{array}{l}3 b \\
4\end{array}$ & $\begin{array}{l}13 \\
18\end{array}$ & $\begin{array}{l}95 \\
80\end{array}$ & $\begin{array}{l}88 \\
13\end{array}$ & 47 & 42 & $66 \bar{a}$ \\
\hline 5 & 14 & 80 & 49 & 25 & & $v$ \\
\hline 6 & 16 & 65 & 18 & 38 & 40 & \\
\hline 7 & 16 & 80 & 116 & & 35 & \\
\hline 8 & 16 & 50 & 51 & 30 & 43 & 57 \\
\hline 9 & 13 & 95 & 67 & 27 & & ᄃ \\
\hline 10 & 12 & 80 & 52 & 48 & 40 & $83 \lesseqgtr$ \\
\hline 11 & 20 & 45 & 23 & 31 & 44 & 59 ه \\
\hline 12 & 15 & 30 & 8 & 140 & 40 & $57 \stackrel{\circ}{3}$ \\
\hline 13 & 13 & 75 & 19 & 56 & 43 & 67 \\
\hline 14 & 19 & 45 & 14 & 43 & 38 & 59 \\
\hline 15 & 15 & 50 & 9 & 39 & 43 & \\
\hline $16 a^{\star}$ & 16 & 80 & 66 & 49 & 39 & $74 \stackrel{\frac{1}{2}}{=}$ \\
\hline $16 \mathrm{~b}$ & 17 & 80 & 41 & 40 & 38 & 68 \\
\hline $17^{\star}$ & 14 & 40 & 13 & 58 & 40 & 68 \\
\hline 18 & 16 & 85 & 27 & 54 & 34 & 70 \\
\hline 19 & 13 & 80 & 84 & 37 & 39 & 83 \\
\hline 20 & 17 & 70 & 41 & 52 & 40 & $63 \frac{0}{\bar{d}}$ \\
\hline
\end{tabular}

Note: The rates are given in the order in which they were measured

$a$ and $b$ refer to the 1st and 2nd studies which were conducted one year apart.

Numbers in parentheses are rates of transport of the same bolus.

$\star$ Studies conducted in the upright position.

tRefers to the transport rate of the least squares fit through all points contributing to the rates in the preceeding brackets. 
and results of serum assay

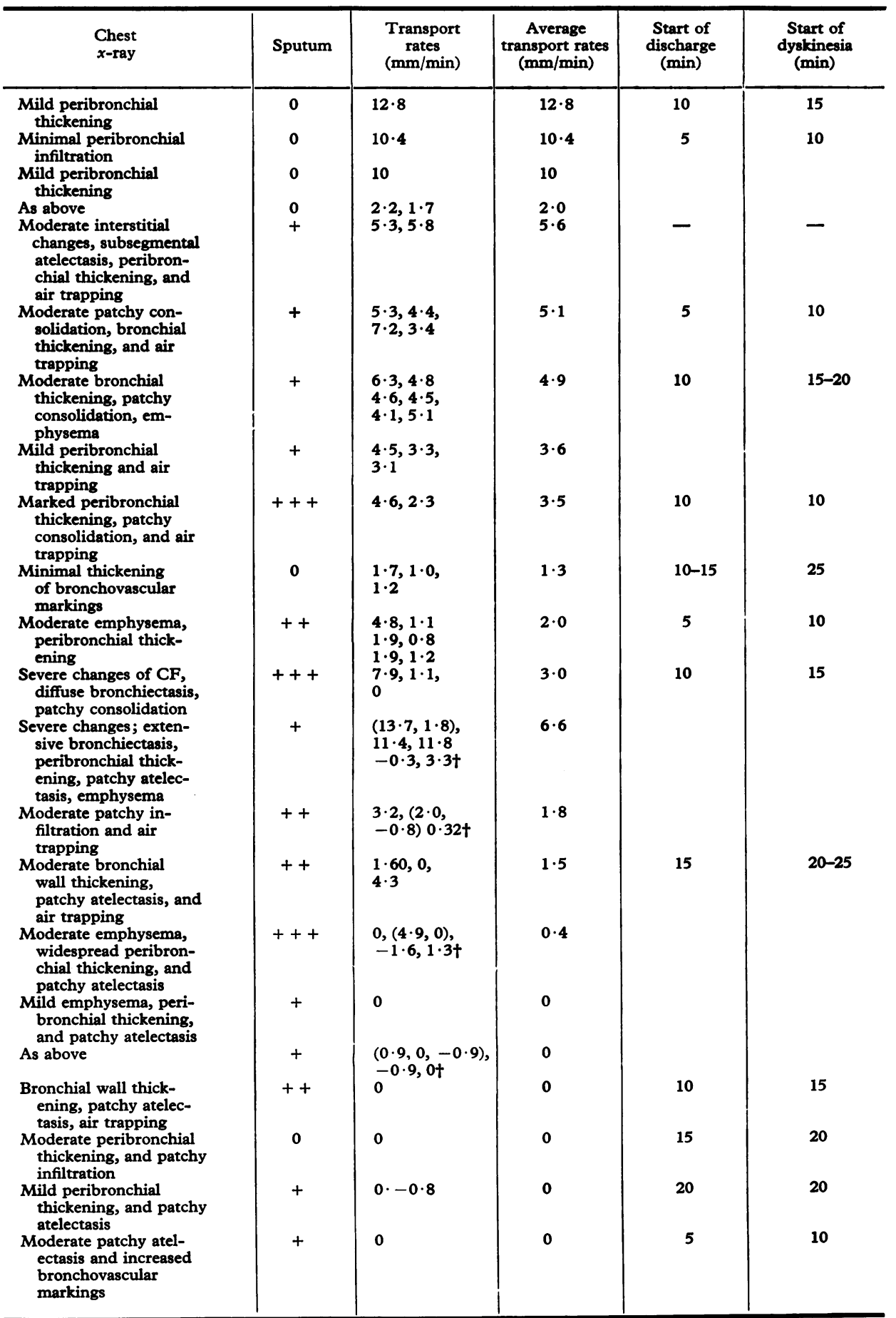


Those patients with a higher arterial oxygen tension and Shwachman score tended to have faster transport rates. Very little correlation could be detected between the rates and the other clinical parameters.

The sera from 14 patients in this study, except from Case 4, gave a positive reaction in the rabbit tracheal assay. After incubation with the CF serum, the discharge of material from the surface of the explant was followed by ciliary dyskinesia and finally complete loss of ciliary activity. The earliest time at which discharge of material was detected was between 5 and 20 minutes after incubation with different sera, whereas the earliest alterations in ciliary activity occurred between 10 and 20 minutes. In general the sera from which the earliest response was observed resulted in a more rapid course of events. There appeared to be a relation between the transport rates and the times at which changes occurred in the assay (Table). With the higher transport rates, there was an early response both in discharge of material $(0 \cdot 2<\mathrm{P}$ $<0 \cdot 3)$ and in ciliary dyskinesia $(0.2<P<0 \cdot 3)$.

\section{Discussion}

The wide variation in tracheal transport rates from normal to abnormal observed in this study is consistent with the wide variation in the severity of the clinical manifestations of this disease. The distribution of mean MTTRs in CF patients in whom no abnormalities in tracheal transport were detected (group 1) is similar to that of a population of healthy adults despite the fact that some of these patients habitually coughed and produced purulent sputum. The mean MTTR of these studies was $6.6 \mathrm{~mm} / \mathrm{min}$ compared to a mean of $4.7 \mathrm{~mm} / \mathrm{min}$ for the healthy adult population (Yeates et al., $1974 \mathrm{~b}$ ) and both populations had a similar range in transport rates $0 \cdot 85-12.8 \mathrm{~mm} / \mathrm{min}$ ). In group 2 were patients who had one or two fast transport rates together with some slow rates (especially Cases 11 and 12). This suggests that their intrinsic rate of transport may have been similar to those of group 1 but was slowed down in certain areas of the trachea either by localized alterations in the ciliated epithelium or in the mucus blanket. In the third group of patients where no positive transport rates were observed, coughing was the major clearance mechanism from the trachea. Cough was not effective as a clearance mechanism in the trachea of healthy adults (Yeates et al., 1975).

As a result of hypersecretion of mucus the mucociliary transport rates in CF patients might be expected to be more rapid than in healthy subjects. This tendency towards rapid MTTRs was evident in Cases 1, 2, 3, 12, and 13. However, Case 9 who had normal pulmonary function and Shwachman score produced no sputum and had a slow transport rate. This was in contrast to Case 18 who had no cough or sputum but had severe lung disease and showed no measurable transport rates.

There were a number of factors which could have contributed to the apparent discrepancy between these results of mucociliary transport and those of Sanchis et al. (1973), Thomson et al. (1974), and Wood et al. (1974). Each group of investigators have used a different tracer of mucociliary activity and therefore may have observed different physiological processes. The results of the first two studies (Sanchis et al., 1973; Thomson et al., 1974) showed an increased tracheobronchial clearance compared to healthy adults which can be attributed not only to normal clearance, but to more proximal deposition of labelled particles, and to coughing.

Using a fibreoptic bronchoscope, Wood et al. (1974) measured the rate of transport of $0.68 \mathrm{~mm}$ teflon discs up the trachea. They reported that CF patients had slower tracheal transport rates than healthy subjects studied by this method. It must be emphasized that the clearance rates in the small airways where the pulmonary disease in CF is first evident cannot be inferred from measurements made in the trachea or large airways. However the results reported here indicate that there is not a general mucociliary defect throughout the respiratory tract in all CF patients.

The fact that most of these studies were conducted in the supine position is not expected to significantly influence the findings. The 2 patients studied twice gave similar results in both the upright and supine positions. The slower transport rate in Case 3 in the supine position, may have been due to the fact that she had recently had tracheitis. A healthy adult subject studied in the supine and upright positions gave similar MTTRs in both studies. The effects of posture on mucociliary transport in health and disease require further investigation.

The results suggest that a higher serum factor activity is associated with faster MTTRs. This is contrary to the expectation that derangement in ciliary activity should result in decreased mucociliary function. In the rabbit trachea explant, the discharge of material preceding ciliary dyskinesia leads to the speculation that this discharge might be related to mucus secretion and be the more important of the two effects. The relevance of the relation between hypersecretion and increased transport rates in the human respiratory tract requires further investigation. 


\section{Mucociliary transport in trachea of patients with cystic fibrosis}

This work was supported by the Canadian Cystic Fibrosis Foundation, the Kinsman Cystic Fibrosis Research Centre, and the Ontario Thoracic Society.

REFERENCES

Camner, P., Jarstrand, C., and Philipson, K. (1973a). Tracheobronchial clearance in patients with influenza. American $R e$ view of Respiratory Disease, 108, 131.

Camner, P., Jarstrand, C., and Philipson, K. (1973b). Tracheobronchial clearance in patients infected with Mycoplasma pneumoniae. Airborne Transmission and Airborne Infection; Concepts and Methods, p. 236 . Ed. by J. F. Hers and K. C. Winkler. Oösthoek, Utrecht.

Charman, J., and Reid, L. (1972). Sputum viscosity in chronic bronchitis, bronchiectasis, asthma and cystic fibrosis. Biorheo$\log y, 9,185$.

Conover, J. H., Bonforte, R. J., Hathaway, P., Paciuc, S., Conod, E. J., Hirschhorn, K., and Kopel, F. B. (1973). Studies on ciliary dyskinesia factor in cystic fibrosis. I. Bioassay and Heterozygote detection in serum. Pediatric Research, 7, 220.

Denton, R. (1960). Bronchial obstruction in cystic fibrosis; rheological factors. Pediatrics, 25, 611.

DuBois, A. B., Botelho, S. Y., Bedell, G. N., Marshall, R., and Comroe Jr., J. H. (1956). A rapid plethysmographic method for measuring thoracic gas volume: a comparsion with a nitrogen washout method for measuring functional residual capacity in normal subiects. Fournal of Clinical Investigation, 35, 322.

Esterly, J. R., and Oppenheimer, E. H. (1968). Observations in cystic fibrosis of the pancreas. III. Pulmonary lesions. Fohns Hopkins Medical fournal, $122,94$.

Feather, E. A., and Russell, G. (1970). Sputum viscosity in cystic fibrosis of the pancreas and other pulmonary diseases. British Fournal of Diseases of the Chest, 64, 192.

Hoorn, B., and Tyrrell, D. A. J. (1966). A new virus cultivated only in organ cultures of human ciliated epithelium. Archiv für die Gesamte Virusforschung, 18, 210.

Lamb, D., and Reid, L. (1972). The tracheobronchial submucosal glands in cystic fibrosis: A qualititative and Quantitative histochemical study. British fournal of Diseases of the Chest, 66, 239.

Matthews, L. W., Spector, S., Lemm, J., and Potter, J. L. (1963). Studies on pulmonary secretions. I. The overall chemical composition of pulmonary secretions from patients with cystic fibrosis, bronchiectasis, and laryngectomy. American Review of Respiratory Diseases, 88, 199.

Potter, J. L., Matthews, L. W., Lemm, J., and Spector, S. (1963). Human pulmonary secretions in health and disease. IV. Studies on mucus in relation to human disease. Annals of New York Academy of Sciences, 106, 692.

Reid, L., and de Haller, R. (1967). The bronchial mucous glandstheir hypertrophy and change in intracellular mucus. Modern Problems in Pediatrics, 10, 195.

Sakakura, T., Sasaki, Y., Hornick, R. B., Togo, Y., Schwartz, A. R., Wagner, H. N., and Proctor, D. F. (1973). Mucociliary function during experimentally induced rhinovirus infection in man. Annals of Otology, Rhinology and Laryngology, 82, 203.

Sanchis, J., Dolovich, M., Rossman, C., Wilson, W., and Newhouse, M. (1973). Pulmonary mucociliary clearance in cystic fibrosis. New England fournal of Medicine, 288, 651.

Spock, A., Heick, H. M. C., and Logan, W. S. (1967). Abnormal serum factor in patients with cystic fibrosis of the pancreas. Pediatric Research, 1, 173.

Sturgess, J. M., and Reid, L. (1969). A new pattern of sputum viscosity. Proceedings of the 5th International Cystic Fibrosis Conference, p. 368 . Ed. by D. Lawson. London.

Thomson, M. L., Pavia, D., Short, M. D., and Norman, A. P. (1974). Lung clearance in two patients with cystic fibrosis. New England fournal of Medicine, 289, 749.

Wood, R. E., Wanner, A., and Hirsch, J. A. (1974). Measurement of mucociliary airway clearance in patients with cystic fibrosis and its stimulation by terbutaline. Pediatric Research, 8, 471.

Yeates, D. B., Warbick, A., and Aspin, N. (1974a). Production of $99 \mathrm{Tcm}$ labelled albumin microspheres for lung clearance studies and inhalation scanning. International fournal of Applied Radiation and Isotopes, 25, 578.

Yeates, D. B., Aspin, N., Levison, H., and Bryan, A. C. (1974b) Measurements of mucociliary tracheal transport rates in man. Federation Proceedings, 33, 365.

Yeates, D. B., Aspin, N., Levison, H., Jones, M. T., and Bryan, A. C. (1975). Mucociliary tracheal transport rates in man. Fournal of Applied Physiology, 39, 487

Zuelzer, W. W., and Newton, W. A. (1949). The pathogenesis of fibrocystic disease of the pancreas. Pediatrics, 4, 53.

Correspondence to Dr. N. Aspin, Research Institute, Hospital for Sick Children, 555 University Avenue, Toronto, Ontario, Canada M5G 1 X8. 